\title{
Noninvasive method to validate the variability of blood pressure during arrhythmias
}

\author{
Tomonori Watanabe $^{1} \cdot$ Satoshi Hoshide ${ }^{1} \cdot$ Kazuomi Kario $^{1}$
}

Received: 12 November 2021 / Revised: 23 November 2021 / Accepted: 23 November 2021 / Published online: 27 December 2021

(c) The Japanese Society of Hypertension 2021

For the management of hypertension, cuff-based noninvasive blood pressure (BP) measurement has established accuracy and is widely used in various settings (e.g., office, home, and ambulatory conditions). All BP values measured by this technique provide both the information necessary for an accurate diagnosis of hypertension and prognostic power for cardiovascular disease (CVD) events, and the reduction of high BP levels leads to the prevention of CVD events [1]. In addition, various BP variability parameters, such as short-, mid-, and long-term variability assessed by office BP, home BP, and ambulatory BP monitoring, are associated with the progression of target organ damage and a risk of CVD events, independent of the patients' average $\mathrm{BP}$ values [2-4]. However, the evidence underlying these associations was obtained in studies of populations without arrhythmia or in investigations that ignored the contribution of arrythmias, since the device used for the BP measurements had been validated only under sinus rhythm.

Each BP value is obtained from the pulse wave from the time point at which the cuff is inflated to the time it is deflated. The values may be affected by the temporal variation and magnitude variation of the pulse wave associated with the irregularity of the heartbeats. The cardiac cycle consists of systolic and diastolic phases, and if ventricular contractility is normal, circulatory dynamics are regulated by left ventricular (LV) ejection during the systolic phase and LV filling during the diastolic phase, in addition to vascular elasticity. Fluctuations in heart rate affect LV stroke volume. Moreover, ventricular arrhythmias are prone to decrease BP because of the loss of atrioventricular (AV) contractile linking by antegrade AV node conduction.

Kazuomi Kario

kkario@jichi.ac.jp

1 Division of Cardiovascular Medicine, Department of Internal Medicine, Jichi Medical University, Tochigi, Japan
The length of time from the QRS complex in electrocardiography monitoring to the maximum pulse wave in the peripheral arteries was defined as the conventional pulse transit time (cPTT) [5, 6], and this time variable has been used in a novel cuffless form of continuous BP monitoring. Miao et al. reported a parameter that can be used for accurate assessment of $\mathrm{BP}$ variability during ventricular arrhythmia based on the concept of the cPTT, and they reported that adjusted PTT $=\mathrm{CPTT}^{2} / \mathrm{R}-\mathrm{R}$ was calculated from the R-R interval of the cardiac cycle during ventricular arrhythmias [7]. In that study, there was a strong negative correlation between the adjusted PTT and the BP assessed by an invasive method during ventricular arrhythmia compared to CPTT. It is assumed that premature ventricular complex and ventricular tachycardia (VT) with a short coupled period results in insufficient LV filling and consequently a decrease in the LV stroke volume. The adjusted PTT appears to be an index that emphasizes the prolongation of the cPTT and decreased BP along with ventricular arrhythmias with a short coupled period. Miao et al. demonstrated that the adjusted PTT is useful as an index to evaluate BP during ventricular arrhythmia. However, there are several limitations regarding the adjusted PTT to consider.

First, the adjusted PTT does not take the variation of LV contractility into account. The lowered LV contraction could decrease the magnitude of the pulse wave, which results in decreased BP values and an increased cPTT. Figure 1 shows three representative cases of catheter ablation for VT, including invasive BP measurement during VT. Each patient's case has a different etiology and illustrates the impact of LV contractility and arterial elasticity on BP during VT.

Case 1, with a normal LVEF and normal arterial elasticity, had a slight decrease in systolic BP during VT, but her pulse pressure was maintained (Fig. 1A). In contrast, in Case 2 with a reduced LVEF and normal arterial elasticity, the systolic BP and pulse pressure strikingly decreased during VT even though the cycle length of the tachycardia was longer than in the other cases (Fig. 1B). 
(A) Case 1. 39 y.o Famale Normal LVEF and Normal arterial elasticity VT: TCL $300 \mathrm{~ms}$ : VT rate $200 \mathrm{bpm}$

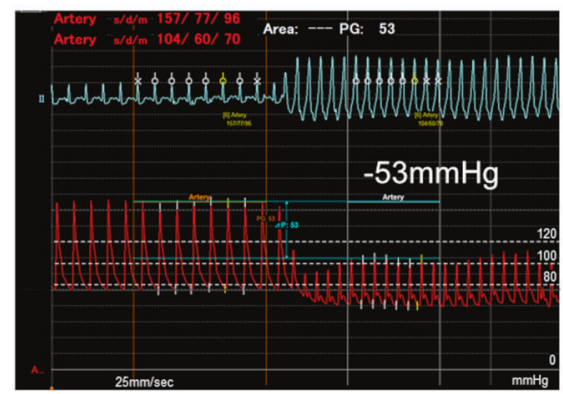

(B) Case 2.60 y.o Male Reduced LVEF and Normal arterial elasticity VT: TCL $430 \mathrm{~ms}$ : VT rate $140 \mathrm{bpm}$
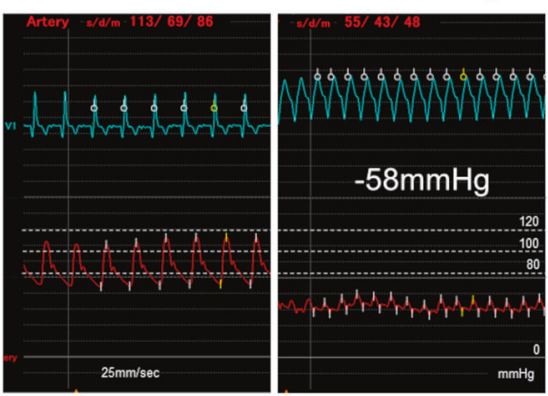

(C) Case 3. 84 y.o Male Normal LVEF and Decreased arterial elasticity VT: TCL $300 \mathrm{~ms}$ : VT rate $200 \mathrm{bpm}$

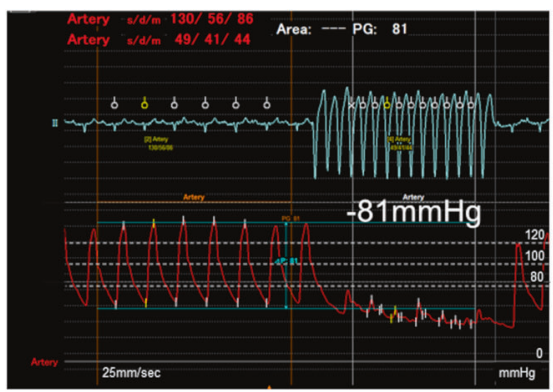

A second consideration is that systemic arterial elasticity should also be taken into account. Increased arterial stiffness may be an important factor for the definition of cPTT because pulse wave velocity, which is the index of arterial stiffness, is included in the formula of the cPTT.

In Case 3 with a normal LVEF and decreased arterial elasticity (Fig. 1C), LV systolic function was preserved, but his systolic BP and pulse pressure were markedly decreased during VT, even though the cycle length of the tachycardia was similar to that of Case 1. Case 3 had preserved LV systolic function, but he had impaired LV relaxation abnormalities related to decreased arterial elasticity and an increased LV mass volume, leading to a decreased stroke volume.
Fig. 1 The patients' characteristics. A Case 1 with normal LVEF and normal arterial elasticity. A 39-year-old female, no other etiology. VT: TCL 300, ms: VT rate 200 bpm. TTE: LVEF 70\%, E/e' $=8.4$, E/A = $2.15, \mathrm{LVMI}=48 \mathrm{~g} / \mathrm{m}^{2}$. Stroke volume index $40.2 \mathrm{ml} / \mathrm{m}^{2}$, LAVI 33.9 $\mathrm{ml} / \mathrm{m}^{2}$. baPWV: unknown data, possible normal. B Case 2 with reduced LVEF and normal arterial elasticity. A 60-year-old male, OMI, postPCI, LV apical aneurysm, DM, DLP. VT: TCL $430 \mathrm{~ms}$, VT rate $140 \mathrm{bpm}$. TTE: $\mathrm{LVEF} 32 \%, \mathrm{E} / \mathrm{e}^{\prime}=7.1, \mathrm{E} / \mathrm{A}=0.69, \mathrm{LVMI}=226$ $\mathrm{g} / \mathrm{m}^{2}$, LVH. Stroke volume index $57.8 \mathrm{ml} / \mathrm{m}^{2}$, LAVI $62.2 \mathrm{ml} / \mathrm{m}^{2}$. baPWV: right 1346, left 1363. C Case 3 with normal LVEF and reduced arterial elasticity. An 84-year-old male, IHD, postPCI, HTN, DM, CKD grade 5, DLP, HUA, prior CI. VT: TCL $300 \mathrm{~ms}$, VT rate 200 bpm. TTE: $\mathrm{LVEF} 63 \%, \mathrm{E} / \mathrm{e}^{\prime}=7.8, \mathrm{E} / \mathrm{A}=0.60, \mathrm{LVMI}=125 \mathrm{~g} /$ $\mathrm{m}^{2}$, LVH. Stroke volume index $43.4 \mathrm{ml} / \mathrm{m}^{2}$, LAVI $42.3 \mathrm{ml} / \mathrm{m}^{2}$. baPWV: right 3004 , left 2755 . CI cerebral infarction, CKD chronic kidney disease, DLP dyslipidemia, DM diabetes mellitus, HTN hypertension, HUA hyperuricemia, IHD ischemic heart disease, LAVI left atrial volume index, LVEF left ventricular ejection fraction, LVH left ventricular hypertrophy, LVMI left ventricular mass index, OMI old myocardial infarction, PCI percutaneous coronary intervention, PWV pulse wave velocity, TCL tachycardia cycle length, TTE transthoracic echocardiography, VT ventricular tachycardia.

There are thus multiple factors (such as LV contractility including both systolic and diastolic function and arterial elasticity) that affect BP variability during arrhythmias, in addition to the irregularity of heart rate.

Third, the consistency between the adjusted PTT parameter and BP levels measured by noninvasive BP monitoring has not been validated. However, this issue is applicable for not only adjusted PTT but also other cuffless wearable BP monitoring device methods that include cPTT, and the assessment of the accuracy of these BP measurement devices requires the use of a validation protocol.

Although atrial fibrillation (AF) does not cause hemodynamic changes compared to ventricular arrhythmia, the number of patients with AF has been increasing, and $\mathrm{AF}$ is one of the arrhythmias for which hypertension is a major complication; in addition, AF is associated with a risk of thrombotic events and incident heart failure [8,9]. We reported the specific shape of the oscillometric curve and arrhythmia characteristics due to the intracuff pressure waveform during cuff contraction in oscillometric BP measurement with a new BP monitoring device [10]. It is also important to assess the impact of heart rate variability on $\mathrm{BP}$ variability during arrhythmias by determining the patterns of the oscillometric curve and intracuff pressure waveform. Especially in patients with AF, it is known that $\mathrm{BP}$ variability is caused by fluctuations in the heart rate and diastolic phase with a loss of atrial kick and LV filling, and $\mathrm{BP}$ variability is induced by variability in the cardiac output, resulting in a "pulse deficit." Accurate assessments of BP indices in patients with $\mathrm{AF}$ along with irregularity in the $\mathrm{R}-\mathrm{R}$ interval must also take the heart rate into account, and it is necessary to verify the accuracy of the cPTT during AF. 
The current validated device for BP measurements was derived from the cuff-based method. However, it is not possible to perform continuous BP measurements with this device, which may cause a burden for patients. With the further development of noninvasive cuffless BP measurement and its validation, new information for the control of BP under various conditions, including arrhythmia, could be obtained, although this presents a significant challenge $[11,12]$.

\section{Compliance with ethical standards}

Conflict of interest The authors declare no competing interests.

Publisher's note Springer Nature remains neutral with regard to jurisdictional claims in published maps and institutional affiliations.

\section{References}

1. Umemura S, Arima H, Arima S, Asayama K, Dohi Y, Hirooka Y, et al. The Japanese Society of Hypertension Guidelines for the Management of Hypertension (JSH 2019). Hypertens Res. 2019;42:1235-481.

2. Kario K, Chirinos JA, Townsend RR, Weber MA, Scuteri A, Avolio A, et al. Systemic hemodynamic atherothrombotic syndrome (SHATS) - coupling vascular disease and blood pressure variability: proposed concept from pulse of Asia. Prog Cardiovasc Dis. 2020;63:22-32.

3. Hoshide S. Clinical implication of visit-to-visit blood pressure variability. Hypertens Res. 2018;41:993-9.
4. Hoshide S, Yano Y, Mizuno H, Kanegae H, Kario K. Day-by-day variability of home blood pressure and incident cardiovascular disease in clinical practice: The J-HOP Study (Japan Morning Surge-Home Blood Pressure). Hypertension. 2018;71:177-84.

5. Mukkamala R, Hahn JO, Inan OT, Mestha LK, Kim CS, Töreyin $\mathrm{H}$, et al. Toward ubiquitous blood pressure monitoring via pulse transit time: theory and practice. IEEE Trans Biomed Eng. 2015;62:1879-901.

6. Block RC, Yavarimanesh M, Natarajan K, Carek A, Mousavi A, Chandrasekhar A, et al. Conventional pulse transit times as markers of blood pressure changes in humans. Sci Rep. 2020;10:16373.

7. Miao F, Zhou B, Liu Z, Wen B, Li Y, Tang M. Using noninvasive adjusted pulse transit time for tracking beat-to-beat systolic blood pressure during ventricular arrhythmias. Hypertens Res. 2021. https://doi.org/10.1038/s41440-021-00795-y2.

8. Gage BF, Waterman AD, Shannon W, Boechler M, Rich MW, Radford MJ. Validation of clinical classification schemes for predicting stroke: results from the National Registry of Atrial Fibrillation. JAMA. 2001;285:2864-70.

9. Bassand JP, Accetta G, Camm AJ, Cools F, Fitzmaurice DA, Fox $\mathrm{KA}$, et al. Two-year outcomes of patients with newly diagnosed atrial fibrillation: results from GARFIELD-AF. Eur Heart J. 2016;37:2882-9.

10. Watanabe T, Tomitani N, Kario K. Perspectives on an ambulatory blood pressure monitoring device with novel technology for pulse waveform analysis to detect arrhythmias. J Clin Hypertens (Greenwich). 2020;22:1525-9.

11. Yang S, Morgan SP, Cho SY, Correia R, Wen L, Zhang Y. Noninvasive cuff-less blood pressure machine learning algorithm using photoplethysmography and prior physiological data. Blood Press Monit. 2021;26:312-20.

12. Koshimizu H, Kojima R, Okuno Y. Future possibilities for artificial intelligence in the practical management of hypertension. Hypertens Res. 2020;43:1327-37. 